Il comprend 14 panneaux de $1 \mathrm{~m}$. de largeur, montés sur une charpente métallique.

Les 5 premiers panneaux en marbre blanc, correspondent chacun à un alternateur et portent un ampèremètre placé sur l'une des phases, un ampèremètre d'excitation, des lampes témoins, le levier de commande d'un interupteur automatique à action difterée, et le volant de manouvro du rhéostat d'excitation. Le panneau suivant comporte les appareils de couplage, lampes de phase, voltmètres, etc. Sur ce panneau se trouvent deux volants qui permettent, soit de manœuvrer toutes ensemble les commandes des rhéostats d'excitation par l'un ou l'autre volant, soil de former deux groupes distincts.

Vieunent ensuite 2 panneaux pour les excitatrices, puis 3 panneaux pour le couplage des transformateurs (les deux premiers correspondent chacun à deux transformateurs). Le panneau qui suit comporte 2 voltmètres donnant la tension des ligues. Les deux derniers panneaux, dont un pour chaque ligne, compoltent trois ampèremètres un waltmètre enregistreur, un compteur, et la commande des interruptcurs de ligne.

Il y a deux sćries de barres omnibus à haute tension permettant d'alimenter séparément, ou en parallèle. les deux lignes avec un transformateur quelconque. Il existe éralement des barres omnibus à basse tension, mais elles ne servent pas qu'accidentellcment, chaque alternateur débitant toujour's en temps normal sur chacue transformateur.

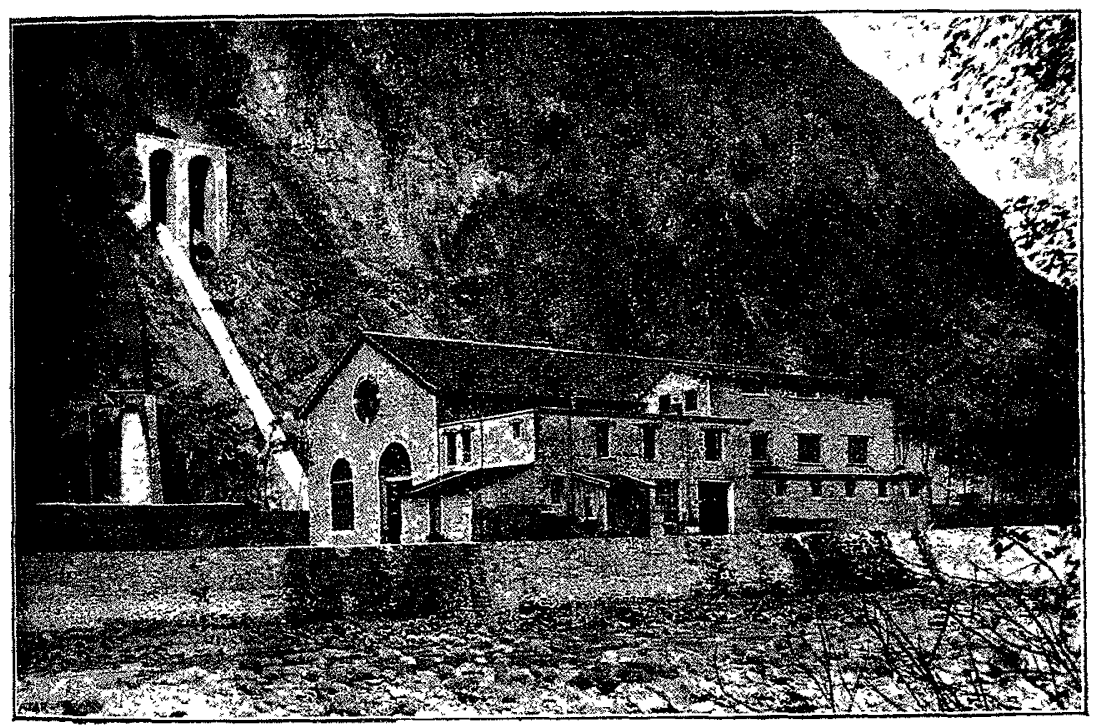

Fia. 10. - Vue générale de l'usine de Sj́chilienno.

Les interrupteurs de ligne sont établis pour une tension do 45000 volts. 11 s sont à rupture dans lihuile, et sont actionnés par une commande electrique.

Les décliarseurs sont des parafoudres à cornes ordinairos, et sont mis à la terre par l'intermédiaire de résistances liquides. Les limiteurs de tension sont constitués par des filets d'eau, provenant d'entonnoirs en communication avec chacun des fils de ligne, qui tombent dans une cuve mise à la terre.

Lignes. - Deux lignes, composées chacune de 3 fils de cuivre, de $7 \mathrm{~mm}$ de diamètre, distants de $1 \mathrm{~m}$. les uns des autres, partent de l'usine de Séchilienne. Ces deux lignes sont montées sur les mêmes poteaux. Ceux-ci sont métalliques, et ont $10 \mathrm{~m}$. de hauteur au-dessus du sol. Ils sont formés de 4 cornières, sensiblement verticales, assemblées au moyen d'entretoises. Ils ont été construits par la maison Joya. Les isolateurs sont placés sur des traverses en bois formant un cadre à la partie supérieure du poteau.

Ces deux lignes vont se réunir, à Saint-Georges-deCommiers, à colles qui viennent de l'usine d'Avignonnet, pour alimenter le vaste réseau de la Société Grenobloise de Force et Lumière, qui fournit en énergie électrique, non seulement la rogion si industrielle comprise entre Grenoble et Lyon, en passant par Voiron, Bourgoin et Vienne, mais encore-Annonay et ses environs. Nous reviendrons d'ailleurs ultérieurement sur la description de cet important réseau de distribution.

II. Belletet.

\section{LES PHÉNOMÈNES VOLCANIQUES LEURS CAUSES. - LEURS EFFETS}

(SUITE)

\section{$\S$ II. - Causes des manitestations volcaniques.}

Les idées les plus bizarres ont été formulées pour expliquer les phénomènes volcaniques. Aussi, avant d'indiquer quelles sont leurs véritables causes; mentionnons, dans un intérêt à la fois scientifique et historique, quelques-unes des hypothèses qui ont été émises pour les expliquer. Toutes ces hypothèses ont un caractère commun, celui de donner pour point de départ aux phénomènes volcaniques une action chimique dont le premier effet est un dégagement de chaleur. Ainsi que Humboldt l'a fait remarquer, suivant les phases diverses que les sciences chimiques ont parcourues, ces phénomènes_ont été altribués successivement au bitume, aux pyrites, à un mélange humide de fer et de soufre réduits en poussiere.

Les schistes bitumineux, les lignites, certaines houilles, renferment des pyrites qui, une fois mises en contact ayec l'eau superficielie ou atmosphérique, se transforment en sulfate de fer. Cette transformation détermine la production d'une certaine quantité de chaleur, accuséc par la production d'un certain volume de vapcur d'eau qui se dégage des amas de débris accumulés autour des exploitations. Quelquefois les couches de houille ou de lignite s'enflamment spontanément sous l'influence de causes que nous neconnaissons pas d'une manière exacte. Celle inflammation spontanée peut être produite, tantôt par l'oxydation des pyrites placées dans le voisinage dinfiltrations d'eau, tantôt par la combustion du grisou. Quoiqu'il en soit, ces inflammations spontanées allument des incendies souterrains qui durent pendant un temps plus ou moins long, et que Werner appelait des pseudo. yolcans.

La mine de Revaux, dans le bassin de Saint-Elienne, peut être citée comre une de celles qui présentent des traces d'ignilion. D'après A. Burat, sous une couche de fer carbonaté, une couche de houille a été en grande partie changée en coke; toutes les couches voisines de celles ci paraissent avoir éprouvé aussi une très forte calcination; elles sont rouges et moins dures; le sulfure de fer y a éprouvé une forte sublimation, par suite de laquelle il s'est formé du soufre natif et des veines de sulfate de chaux cristallisé.

Quant à l'hypothèse basée sur le mélange de soufre et de fer, elle repose sur cette expérience de Lémery; tout le monde connait cette réaction chimique dans laquelle on produit une sort de volcan en miniature en mélangeant, dans une cavité creusée dans le sol, du soufre et de la limaille de fer humectée d'eau. Au bout de quelque temps, il se produit un jet comtinu de vapeur d'eau qui s'échappe pendant un temps assez long de l'extrémité supérieure. Mais ca n'est là qu'une expérience de laboratoire, et il y a lieu de s'étonner qu'elle ait pu paraîre suffisante à certains esprits, même il y a 50 ans, pour expliquer le phénomène grandiose d'une éruption volcanique avec émission de laves et projection de scories.

C'est de même que Buffon, frappé de la situation des volcans italiens au voisinage de la mer, pensait que les éruptions du 
Vésuve et de l'Etna résultaient du choc des vagues furieuses contre les amas de pyrites et le charbon aciumulés à l'intérieur de ces montagnes. A la suite de Buffon et de Lémery, Werner, l'illustre chef de ce qu'on a appelé l'Ecole des Neptunistes, ne voyait dans chaque éruption qu'un embrasement des couches de houllle au contact des eaux de pénétration de la surface.

Mais Werner ne connaissait les volcans que pour en avoir entendu causer : il ne les avait jamais étudiés de près, et cela le rendait excusable. Aujourd'hui, c'est avec une pleine connaissance de la multiplicité des phénomèmes éruptifs qu'un professeur de Hall, auteur d'unlivre récent, vient de ressusciter ceıte doctrine. Même il la juge suffisante pour expliquer les dislocations de l'écorce et la production, non seulement des tremblements de terre, mais des plus hautes chaines de montagnes. L'action de l'air et de l'eau de mer, arrivant par des fissures à portée de masses combustibles qui ne demandent qu'à s'enflammer, tel devrait être l'unique principe des transformations de l'écorce. Ainsi donc, selon cette théorie, il faut convenir qu'au-dessous de chaque volcan existe une provision locale de matières combustibles, suffisante pour alimenter son activité.

Pour renverser cette hypothèse, il suffit de considérer l'importance intrinsèque des éruptions. Depuis les temps historiques, et mêmo bien avant, cortains volcans tels que l'Eına et, mieux encore, le Manna-Loa des îles Sandwich, n'ont cessé de vomir des coulées dont le volume se compte chaque fois par plusieurs millions de mètres cubes (1). Un mètre cube de roche, pour s'élever à une température voisine de $2000^{\circ}$, exige 1250000 calories, soit la chaleur engendrée par la combustion totale de I 80 kilogrammes de houille, lesquels représentente 14 centièmes de mètre cube. Une coulée réclame donc la disparition d'une couche de combustible ayant les 44 centiemes de son volume. Rappelons maintenant que la seule éruption de 1836, aux îles Sandwich, a fait naître une coulée de soo kilomètres de longueur sur 4800 mètres de largeur moyenne, avec une épaisseur qui, par endroit, atteignait roo mètres; qu'en ı 880 , le même volcan a donné issue à une coulée de plus de 60 kilomè̀tres; enfin que la masse entière de la montagne, depuis le niveau de la mer, et au-dessous jusqu'à +200 mètres d'altitudé, est entièrement constituée par des laves. Quel est donc le bassin houlller, si riche qu'on le suppose, capable de suffire à une pareille consommation?

Car, il faut le remarquer, il s'dgit là d'un phénomène essentiellement local: c'est juste au-dessous du volcan que doivent se trouver, d'une part la provision de combustible nécessaire, de l'autre la roche destinée par sa fusion à engendrer la chaleur. Non sculement de telles accumulations de charbon minéral dépassent absolument tout ce qui est connu jusqu'ici, mais la sortic de pareilles masses ne pourrait manquer de faire naître un vide correspondant et, au lieu de montagnes de laves comme le Manna-Loa, c'est un effondrement qui devrait se produire au centre de la région d'épanchement. On échappe au contraire à une telle conséquence si l'on suppose que les laves viennent d'un foyer situé à une grande distance de la surface. De plus, s'il y avait un rapport de causalité entre l'existence des gisements de combustibles et les manifestatlonsde la chaleurinterne, c'est dıns !e voisinage immédiat des bassins houillers que ces dernières devraient se produire avec le plus d'intensité. Cependant, c'est juste le contraire qui arrive. Il n'existe aucun volcan à proximité des riches bassins de l'Angleterre, de la Belgique ou de l'Amérique. Pour en trouver quelque indice, il faut aller en Saxe, c'est-à-dire justement dans un pays où, postérieurement à leur dépôt, les bassins de charbon de terre ont été traversés par des épanchements de roches ignées. Ainsi, loin que la transformation des gites de combustibles soit la cause du volcanisme, clle en est seulement une conséquence locale.

Cette théorie a du reste déjá été renversée dès 1797 par Dolomieu qui, remarquant que les volcans d'Auvergne reposent sur le granite, en conclut que le foyer n'est pas superficiel, mais quau contraire il a son gisement dans les profondeurs de la

(1) La Formation de l'Ecorce terrestre, par A. DE Lapparevr. terre, et que tous les rolcans communiquent avec la masse interne du globe encore en fusion. Dès lors, l'Ecole des Plulo. nistes, avec Dolomieu, Cordier, Brongniart et Cuvier, tuiom. phait, reconnaissant comme cause première du phénoméne volcanique le feu central. A son tour, cette théorie subit l'assaut des objections, et fut remplacée par une théorje plus éclectique qui, prenant dans chacune des manières de voir les parcelles de vérité qui s'y rencontrent, s'efforça de les grouper et de les adapter aux aspects multiples de ces phénomènes.

Les disciples de cette théorie, dont les plus célèbres furent Bunsen, $\mathrm{Ch}$. Sainte-Claire-Deville, Fauqué, font intervenir la fois les flota de la mer et le feu central. "Si l'on adme l'existence d'une couche de matières en fusion étendue au-des.

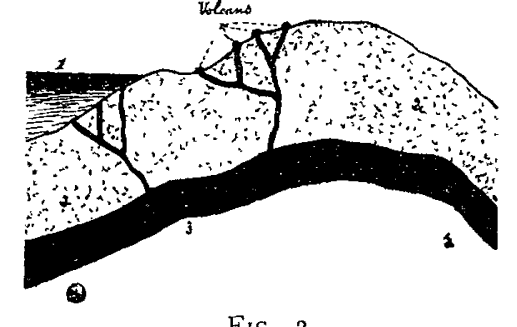

FIG 2 I. Mers. interne du globe.

2. Ecorce solide

3 . Noyau igne.
Action des Eaux marines sur la masse sous de l'écorce terrestre et pénétrant dans ses antfractuosités, dit M. Foy. qué, et si l'on suppose (fig. 2) des infiltrations de l'eau de la mer arrivant jusqu'au contacı du liquide incandescent, toutes les manifestations volcani. ques s'expliquent avec is plus grande facilité s. A l'appui de celte théorie M. Fouqué fait remar. quer que les gaz rejetés par certaius volcans ont la même composition, et sont dans les mêmes proporticns qua les substances contenues dans l'eau de mer. Mais, d'autre part, si cette explication est satisfaisante pout les volcans situés au voisinage de la mer, comment interpréter l'activité des volcans de la Mandchourie, situés à goo kilome. tres de toute masse d'eau?

Quoi qu'il en soit de ces théories, il est certain que la chaleur interne du globe permet seule d'expliquer les phénomènes vol. caniques; s'il n'existait à la surface du globe qu'un petit nom: bre de volcans, ou si les produits épanchés par chacun d'eux n'avaient qu'une importance restreinte, on pourrait être tenté d'y voir des manifestations tout à fait locales, dues à des réac" tions chimiques s'accomplissant dans l'intérieur de l'écorce terrestre. L'intlammation spontanée de combustibles minéraux, la combinaison du soufre avec le fer sous l'influence de l'humi: dité, pourrait provoquer ça et là des incendies locaux. Mais de tels phénomènes seraient forcément très limités, et comme toute combustion active cxige le concours de l'oxygène, de grands embrasements ne pourraient s'accomplir dans les profondeurs du sol où l'air extérieur n'a pas d'accès.

D'autre part, quand on voit qu'un seul volcan, comme celui de l'ile Sandwich, a pu construire avec le temps une accumula. tion de coulées de laves dont le volume đépasse certainement I 000 kilomètres cubes, on se rend compte qu'aucun phéno. mène de combustion interne et locale n'est en état de produire la quantité de chaleur nécessaire pour un pareil résultat. Enlin, la répartition même des volcans invite à leur assigner une causé générale; le pourtour de l'océan Pacifique est jalonné par une ceinture presque ininterrompue de volcans actifs ou éteints depuis peu. Dans l'intérieur de cet immense océan, dontla superficie équivant au tiers de la surface totale du globe, il n'est pas une $\hat{l}$ le qui ne doive sa naissance aux forces volcaniques; don ces forces sont à l'œuvre au moins sur toute cette étendue, et il serait puéril de croire que les volcans se localisent exclusivement au-dessous des points où elles se manitestent par des éruptions proprement dites.

Nous voilà donc arrivés à la véritable cause des phénomènes que nous étudions. En cffet, il est vraisemblable d'admettle avec de Lapparent que les volcans s'alimentent tous à un foyer commun, à une nappe continue, mais cela à des niveaux diflé. rents, à des parties plus ou moins profondes.

L'intérieur du globe, nous l'avons vu, est à une très haute température; son foyer est composé d'une masse ignée, mais non d'une masse liquide. C'est qu'en effet, plus nous descen. 
dons dans l'intérieur de la terre, et plus les couches extérieures superposées exercent une pression considérable surla base. Or, la pression, en rapprochant les molécules des corps, a pour effet de contrarier leur liquéfaction. Il est donc parfaitement possible que le noyau, tout en possédant une température qui le rendrait liquide s'il ne portait d'autre poids que celui de l'atmosphère terrestre, soit pratiquement solide, c'est-à-dire dans le même état de cohésion qu'un corps solide.

Mais cela n'a qu'un faible retentissement sur les phénomènes que nous étudions; en effet, qu'une cause extérieure vienne a supprimer ou à réduire la pression que supporte le noyau ignć, immédiatement la liquidité se manifestera. Or, une écorce de 60 kilomètres au plus d'épaisseur, recouvrant un noyau de plus de 6000 kilomètres, et refroidie elle-même à la surface par le contact avec l'espace extérieur, ne saurait se maintenir sans deformations que si elle était absolument homogène, et si les conditions de son support demeuraient invariables. Il n'en est pas ainsi: d'abord l'écorce n'est pas homogène; ensuite son support perd de la chaleur ave le temps, ce qui l'oblige à se contracter; alors l'écorce, devenue trop ample, est contrainte, pour rester appliquée contre la parie interne, de se déformer; elle se gauchit, se plisse, s'accidente de bourrelets; et, par endıoits, là où la rigidité des roches est trop grande pour se prêter à la formation d'un pli, l'écorce se brise. Le vide engendré par celte cassure redevenue localement liquide monte dans les fissures.

Elle y monte d'autant mieux que le noyau igné n'est pas, comme on pourrait le croire, un simple bain de métaux et de pierres en fusion; il renferme dans sa masse une grande provision de gaz et de vapeurs, tenus en dissolution depuis le moment où notre terre était à l'état de nébuleuse, et qui doivent tendre à se degager avec le temps. De la sorte, celte tendance à la sortie des vapeurs serait la cause principale de l'ascension des laves dans les cassures de l'écorce : elle explique de même le cratère généralement d'explosion des appareils volcaniques.

On sait, en effet, que presque tous les volcans consistent en accumulations de matériaux projetes avec violence et retom. bant autour de l'orifice de sortie, de manière à former des amas coniques, où les couches de débris rejetés alternent avec les nappes de laves épanchées. C'est que, on arrivant à la surface, les gaz subissent une diminution de pression; aussi leur force ćlastique les porte-t-elle, à un certain moment, 'à crever la lave, liquide avec laquelle ils montent, et les débris de cette lave emportés avec les gaz, constituent les cendres et les pierres dont la chute accompagne toujours les paroxysmes volcaniques. D'ailleurs la sortie des gaz est plus ou moins violente, suivant que les matières accompagnantes y font plus ou moins obstacle. Il est des volcans, comme celui des îles Sandivich, où la lave est si Huide que jamais elle n'oppose aux gaz de resistance sirieuse. Ceux-ci se dégagent donc tranquillement sans provoquer d'explosions.

All contraire, dans beaucoups lde volcans, comme ceux de la Sonde, en Océanie, et ceux des Antilles, la lave est très peu Huide; elle demeure visqueuse, et forme sur les fissures, par où clle devrait sortir, des tampons à moitié solides. Aussi les gaz sont-ils forcés de s'accumuler parderrière en pression, et quand leur tension est suffisamment forte, ils font sauter l'obstacle en projetant au dehors une pluie de pierres en pleine incandes-

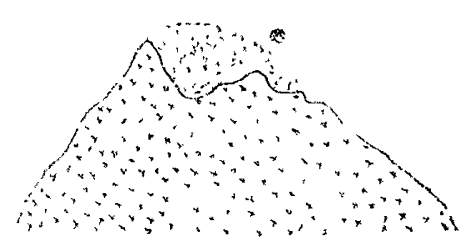

FIG. 3.

Montagne projetée par une éruption. tie partie en pointillé indique la partic de la montagne qui a disparu à la suite de l'explosion.) cence. Une montagne entière, le mont Bandas, au Japon, a été ainsi projetée dans l'aspace (fig. 3) par la force expansive de la vapeur d'eau contenue dans les profondeurs du sol. La muntagne avait fait explosion à la façon d'une gigantesque chaudière. A la place de cette montagne, s'ouvrit un immense gouffre de 3 ooo mètres de longueur,
l'Lina sont intermédiaires entre ces deux extrême, s et c'est pourquoi les phénomènes explosifs y sont en général peu prononcés, ne devenant particulièrement violents que lorsque le volcan a traversé une longue période de repos, ce qui fait que ses orifices naturels se sont encombrés, et qu'il faut un effort special pour les degager.

Ces phénomènes arrivent principalement lorsque les dégagements gazeux se manifestent seuls, et, s'ils sont abondants, la fracture originelle ne suffit pas; en raison de la pression énorme exercée par ces gaz comprimés, elle s'étoile au point de concentration maxima de l'effort; il se produit alors, au centre de l'étoilement, un vaste orifice circulaire, un cratère, four employer l'expression consacrée, par suite de la projection des parties du sol entamé sous ce choc violent. C'est là l'origine de ces cavités circulaires, maintenant occupees par des caux douces, qui forment dans certaines régions ces lacs pittoresques connus sous le nom de cratères-lacs. Le célèbre lac Pavin, en Auvergne, le Gour de Tanczal, près de Manzat, entaillés l'un dans la lave basalique, l'autre dans le granite, en sont de très bons exemples. Ces gouffres lacustres sont surtout connus et nombreux dans l'Eilel (Prusse rhénane), oû on leur a donné le nom bien significatif de maare (gouffre d'eau); des explosions violentes les ont ouverts au travers des schistes dévoniens. Quelques-uns atteignent des dimensions considérables, avec une profondeur de plus de 200 mètres: 1els sont ceux de Gillenferd ct de Laak, qui occupent chacun une sunface de près de 9 kilomètres carrés. M. Vélain a fait remarquer qu'à Nossi-Bé, près de Madagascar, ces cratères-lacs sont disposcis par groupes autour des cônes volcaniques qui se dressent dans les parties centrales de lîle; ils se signalent par la régularité de leur forme circulaire. Les indigènes leur ont donné le nom de lanćlastak, ce qui signifie montagne tombée dans un trou. Occupés maintenant par des eaux d'un bleu d'azur, où vivent des milliers de poissons aux vives couleurs, il constituent lá de véritables aquariums naturels, qui comptent comme une des merveilles de l'ìle.

Les origines de ces carités singulières, entaillées ainsi comme à l'emporte-pièce dans un sol resté horizontal, ontétélongtemps méconnues; on les a attribuces longtempsàdeseffondrements. On sait maintenant que ce sont là des cratères d'explosion, dont la formation, comparable à un coup de mine, doit être rapportéc, comme nous venons de le dire, à l'expansion subitc de masses gazeuses momentanément comprimées. Les iles de la Sunde ont été souvent le théâtre de pareils faits. En 1815 , à Sumatra, le Temboro perdait d'un seul coup 1.600 mètres de sa haucur "Sous ces débris, dont le volume a éte évalué à près de I too kilometres cubes, soil trois fois celui du mont Blanc, dit M. Vélain, la ville de Temboro, située au pied du volcan, a cté ensevelie et 12000 personnes ont péri. L'île de Bornéo, situéc à t fo kilomètres au nord du sićge de l'éruption, lut cnticrement couverte par les cendres qui occasionnirent un immense désastre ».

Mais, fort heureusement, ces grandes explosions sont rares; ordinairement, c'est le sommet seul de l'édifice volcanique qui est emporté, et la montagne reste tronquée. Cu fait s'est préscnté à l'ile Amsterdam, dans l'océan Indien; il a mis fin à une longue période d'activité qui avait donné naissance, à plus de 2000 kilomètres de toute ierre, à un massif volcanique élevé.

(à suivre).

Jean Lscard.

Renargues. - Jans une communcalion falle le 5 mars 1909 it la Sociélé des Ingénieurs Clvils de lrance, M.P.Besson a indiqué que Strult a montré que les roches contenaient toules du rarlium al a cmss l'idée que la chaleur interne de la terre avail pour ortgıne la décomposition du radium. M. Besson estime que le radium ne doll ètre contenu que dans l'écorce (les laves el aulres produit, radioachifs n'élant pas volcanques) el que celle écorce eshlimiléc à une éparsseur de $50 \mathrm{kms}$, épaisseur léjá indiquée pas le prof. Mine en so hasint sur la vitesse de propagation des muveinents sismiques. 